\title{
Double Lung Point Vs X-Ray in Transient Tachypnea of Newborn
}

\author{
Poorvi ${ }^{1}$, Roshan Ann Oommen², Santosh T Soans ${ }^{3}$ \\ ${ }^{1}$ Junior resident, Department of Pediatrics, AJ Institute of medical sciences, Kuntikana, Mangalore, ${ }^{2}$ Professor, Department of Pediatrics, AJ Institute of medical \\ sciences, Kuntikana, Mangalore, ${ }^{3}$ Professor and Head, Department of Pediatrics, AJ Institute of medical sciences, Kuntikana, Mangalore.
}

\section{Abstract}

Background: Transient tachypnea of the newborn (TTNB) is one of the most common causes of perinatal dyspnea and is traditionally diagnosed by chest $\mathrm{x}$-ray. Lung ultrasound is an upcoming tool which is being proved in recent studies to have a better diagnostic capability with the main characteristic feature being the Double Lung Point. Aims \& objectives: To define ultrasonographic appearance of TTNB, evaluate its clinical relevance for early diagnosis and compare the outcome with xray and to assess the diagnostic ability of Double lung point. Subjects and Methods: All newborns presenting with tachypnea within 4 hours of life in $1 \frac{1}{2} 2$ years of study period. (November 2017-May 2019) were enrolled for study. Methods of collection of data: All newborns fulfilling the inclusion criteria were included as study subjects, At same time CHEST XRAY and LUS were done. Statistical analysis: Fisher's exact test was used as test of significance for qualitative data. Continuous data was represented as mean and standard deviation. Mann Whitney U test and Kruskul-wallis test was used for subgroup analysis. P value of $<0.05$ was considered as statistically significant. Result: In study period of $1 \frac{1}{12}$ years 99 cases which were admitted in NICU with respiratory distress meeting the criteria were included. 60 cases were diagnosed to have TTNB, 34 RDS \& 5 Pneumonia. Chest xray and Lung ultrasound (LUS) of these 60 TTNB cases were compared. LUS of TTNB and other cases was also compared for presence of double lung point (DLP). The sensitivity \& specificity of DLP in diagnosis of TTNB was noted to be $68 \%$ \& $100 \%$ respectively with $100 \%$ Positive predictive value. Thus confirming LUS to be a more reliable diagnostic tool than x-ray. Conclusion: Therefore LUS could be used widely in NICU as 1st line of diagnostic medium in diagnosis of various respiratory conditions and early initiation of treatment accordingly.

Keywords: LUS, TTNB, DLP, respiratory distress, X-ray.

Corresponding Author: Dr. Roshan Ann Oommen, Professor, Department of Pediatrics, AJ Institute of medical sciences, Kuntikana, Mangalore.

Email: happypoorvi1993@gmail.com

Received: February 2020

Accepted: March 2020

\section{Introduction}

Transient tachypnea of new born (TTNB) also called as "WET LUNG" is one of the most common causes of perinatal dyspnea.

Transient tachypnea of the newborn (TTNB) is a benign self-limited respiratory distress syndrome of term and late preterm infants related to delayed clearance of lung liquid TTNB can last from 4 to 72 hours after birth. ${ }^{[5]}$

\section{Transient tachypnea of newborn:}

Antenatally, liquid in the distal airway plays a crucial role in fetal lung growth \& development by maintaining the lungs in a distended state which is a potent stimulus for fetal lung growth. Larger liquid volumes provide a greater stimulus for lung growth during gestation. ${ }^{[1]}$

However at birth this interstitial lung fluid should get cleared for entry of air into lung and exchange of gases across alveoli. $^{[2]}$

At birth lung practices this clearance of fluid into surrounding parenchymal tissue within minutes and from there to lymphatics \& blood vessels within 4 to 6 hours of birth. ${ }^{[15]}$ As a result there is effect on breathing and newborns present with distress due to volume overload in lungs.

Hence it is thought to be result from a failure to adequately clear airway liquid after birth and is characterized by tachypnea with signs of mild respiratory distress, including retractions and cyanosis; decreased oxygen saturation is usually alleviated by supplemental oxygen with $\mathrm{FiO} 2<$ $0.04 .^{[11]}$

More common in infants born near term by elective caesarean section (CS) which are not in labour. ${ }^{[3]}$ The most detailed studied theory explaining TTNB is that without the stress of labor, sodium reabsorption is reduced, resulting in airway liquid retention. ${ }^{[4]}$

Delay in this essential process of clearance of fetal lung fluid results in the transient pulmonary edema that characterizes TTNB. Compression of the airways by fluid accumulated in the interstitium can lead to airway obstruction, air trapping, and ventilation-perfusion mismatch. Because newborns usually recover rapidly with short course, a definitive universal pathologic definition is not available. ${ }^{[1]}$

\section{Lung ultrasound:}

TTNB has been always diagnosis of exclusion, with Chest 
Xray being Gold standard was aiding in this diagnosis process.

However few essential diagnoses are missed in which respiratory can also resolve/decrease in time frame of 4 to 72 hours. Hence need for a reliable screening tool/ investigation is needed for effective management of respiratory distress in newborn, thus reducing the stay of newborn in ICU and lesser morbidities

The causes for respiratory distress in newborns range from TTNB, Respiratory distress syndrome (RDS) to sepsis, congenital heart diseases (CHD), pneumonia etc. ${ }^{[16]}$

Respiratory distress after birth may be due to several clinical conditions [e.g. transient tachypnea of the newborn (TTNB), pneumothorax, pneumonia, sepsis, respiratory distress syndrome (RDS), congenital heart disease] that are often difficult to differentiate. At present lung sonography is not routinely included in the diagnostic work-up of these diseases.

Lung ultrasound (LUS) is an easy, reliable, fast tool for screening and diagnosis conditions with minimal or no side effects. Very few studies have been conducted in India pertaining to use of LUS in NICU care.

LUS is accurate and reliable for the early diagnosis of TTNB and is also very useful to easily distinguish TTNB from RDS.

Double lung point in LUS is definitive of TTNB which is not observed in any other condition.

Hence the aim of this study was to define the ultrasonographic appearance of TTNB and evaluate its clinical relevance.

\section{Subjects and Methods}

A prospective time bound study was conducted in neonatal intensive care unit of AJ hospital.All newborns presenting with tachypnea and respiratory distress within 4 hours of life to AJ Hospital, Mangalore during the study period of $11 / 2$ years. (November 2017-May 2019) were enrolled for study Inclusion criteria: All babies presenting with tachypnea \& respiratory distress within 4 hours of life. Exclusion criteria: Newborns presenting after 4 hours of life were excluded from the study. Fisher's exact test was used as test of significance for qualitative data.Continuous data was represented as mean and standard deviation. Mann Whitney $\mathrm{U}$ test and Kruskul-wallis test was used for subgroup analysis.

$\mathrm{P}$ value of $<0.05$ was considered as statistically significant

\section{Methods of collection of data:}

All newborns fulfilling the inclusion criteria were included as study subjects .General physical examination was done and relevant history was noted. In a quiet state, the infants were placed in the supine, lateral, or prone position for the scan. Each lung was divided into 3 regions-anterior, lateral, and posterior regions - by the anterior and posterior axillary line. The probe was perpendicular to the ribs. Each region of both lungs was carefully scanned. At same time CHEST XRAY was taken

\section{Lung ultrasound features}

The superficial layers of the thorax is made of subcutaneous tissue and muscles

Beyond the pleura-lung interface, the lung is air-filled which does not allow further visualization of lung parenchyma in normal conditions. However, due to various respiratory conditions at birth causing the large change in acoustic impedance at the pleura-lung interface results in horizontal artifacts that are seen as a series of echogenic parallel lines equidistant from one another below the pleural line. ${ }^{[16]}$

The upper rib, lower rib and the pleura outline the characteristic "BAT SIGN" On longitudinal scan:

Ribs appear as curvilinear structures associated with posterior acoustic shadowing.

The pleura appears as a echogenic line (pleural line) moving continuously during respiration.

Pleural line: the regular echogenic line under the superficial layers of the thorax moving continuously during respiration, Pleural movement has been described as the 'lung sliding' sign while abnormal pleural lines refer to the pleural line disappearance, thickening, irregularity, or a coarse and indistinct appearance; A-line: a series to echogenic , horizontal, parallel lines equidistant from one another below the pleural line B-lines: also known as ultrasound lung comets, hyper echoic narrow-based artefacts spreading similar to laser rays from the pleural line to the edge of the screen Interstitial syndrome: the presence of more than 3 Blines in every examined area; White lung: defined as the presence of compact B-lines in the 6 areas without horizontal reverberation Lung consolidation: areas of hepatisation with the presence of air bronchograms and/or fluid bronchograms.

Double lung point (DLP) : because of a difference in the severity or nature of the pathological changes in different areas of the lung, a longitudinal scan shows a clear difference between the upper and lower lung fields; this sharp cut-off point between the upper and lower lung fields is known as the DLP Pleural effusion: anechoic-dependent collections limited by the diaphragm and the pleura.

\section{Double lung Point}

Either unilaterally or bilaterally very compact B lines in the in inferior pulmonary fields while in the superior fields the B lines are present but not compact. Thus the border between the inferior pulmonary fields, and the superior ones is termed as the 'double lung point.

\section{Results}

In the study period of $1 \frac{1 / 2}{2}$ years between November 2017 May 2019, we had total 99 cases of respiratory distress 
presenting to AJ hospital which were included in the study after meeting the inclusion criteria .

\section{At the end of course in hospital these cases were given} the following clinical diagnosis:

- Transient tachypnea of newborn

- Respiratory distress syndrome

- Congenital pneumonia

The 60 cases of TTNB were evaluated, detailed history noted and a comparison between LUS and xray done.

Of the 60 cases, 45 were male and 15 were female with gestational age ranging from 32 weeks+ 1 day to 40 weeks + 2 days with mean of 38 weeks +4 days; Higher number noted between 37 weeks to 40 weeks of gestation. 37 newborns delivered via LSCS developed TTNB compared to 23 babies born via vaginal delivery

Table 1: Duration of NICU stay in days

\begin{tabular}{|l|l|l|l|l|}
\hline Cases & Mean & $\begin{array}{l}\text { Standard } \\
\text { deviation }\end{array}$ & Median & $\begin{array}{l}\text { Interquartile } \\
\text { range }\end{array}$ \\
\hline TTNB & 3.56 & 0.97 & 2 & 1.25 \\
\hline
\end{tabular}

[Table 1] shows the average duration of stay of newborns in NICU. No statistical significance noted.

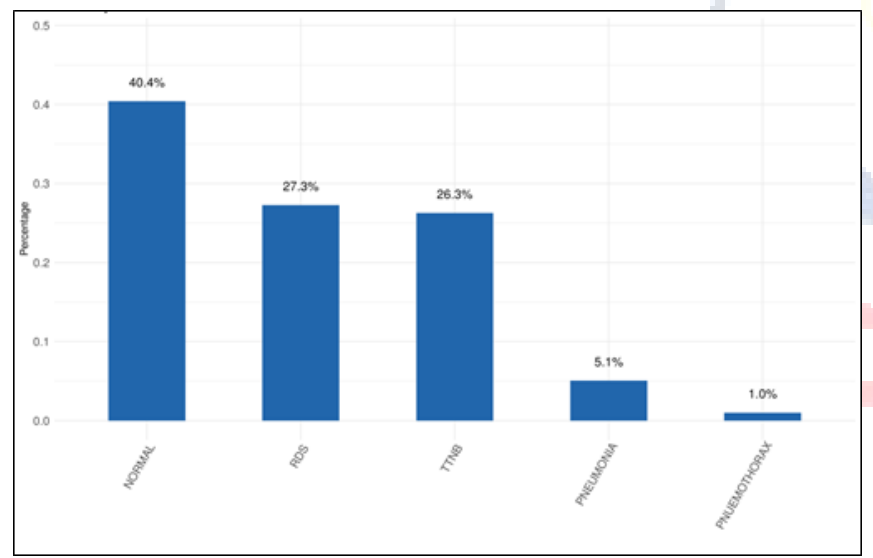

Figure 1: Diagnosis based on X-ray

[Figure 1] shows diagnosis of cases included in study based on X-ray. Out of total no of cases $(n=99), X$-ray was normal in 40 cases, followed by features suggestive of RDS in 27 cases, TTNB in 26 cases.

Table 2: Sensitivity \& specificity of double lung point sign

\begin{tabular}{|l|l|l|l|}
\hline Double Lung Point & TTNB & $\begin{array}{l}\text { Other } \\
\text { Conditions }\end{array}$ & Total \\
\hline Present & 41 & 0 & 41 \\
\hline Absent & 19 & 39 & 58 \\
\hline Total & 60 & 39 & 99 \\
\hline
\end{tabular}

Sensitivity: $68 \%$, Specificity: $100 \%$

Positive predictive value: $100 \%$, Negative predictive value: $39 \%$

[Table $2 \& 3$ ] depict the specificity of double lung point (DLP) sign in cases of TTNB. It is not a feature of other diseases unlike rest of the ultrasound findings such as interstitial lung syndrome, pleural line abnormalities, consolidation, and disappearance of $\mathrm{A}$ line can be found in various causes of respiratory distress. Comparison done by Fisher's exact test (P value of 0.002 which is significant).

Table 3: Ultrasound findings in different cases

\begin{tabular}{|l|l|l|l|l|}
\hline Usg findings & $\begin{array}{l}\text { Ttnb } \\
(\mathbf{6 0 , \%})\end{array}$ & $\begin{array}{l}\text { Rds } \\
(\mathbf{3 4 , \% )}\end{array}$ & $\begin{array}{l}\text { Pneumonia } \\
\mathbf{( 5 , \% )}\end{array}$ & $\begin{array}{l}\mathbf{P} \\
\text { value }\end{array}$ \\
\hline $\begin{array}{l}\text { Double lung } \\
\text { point }\end{array}$ & $41(68)$ & $0(0)$ & $0(0)$ & 0.002 \\
\hline Consolidation & $0(0)$ & $30(88)$ & $5(100)$ & 0.002 \\
\hline $\begin{array}{l}\text { Pleural line } \\
\text { bbnormalities }\end{array}$ & $60(100)$ & $34(100)$ & $5(100)$ & 0.002 \\
\hline $\begin{array}{l}\text { Interstitial } \\
\text { syndrome }\end{array}$ & $60(100)$ & $34(100)$ & $5(100)$ & 0.002 \\
\hline $\begin{array}{l}\text { A line } \\
\text { disappearance }\end{array}$ & $60(100)$ & $34(100)$ & $5(100)$ & 0.002 \\
\hline
\end{tabular}

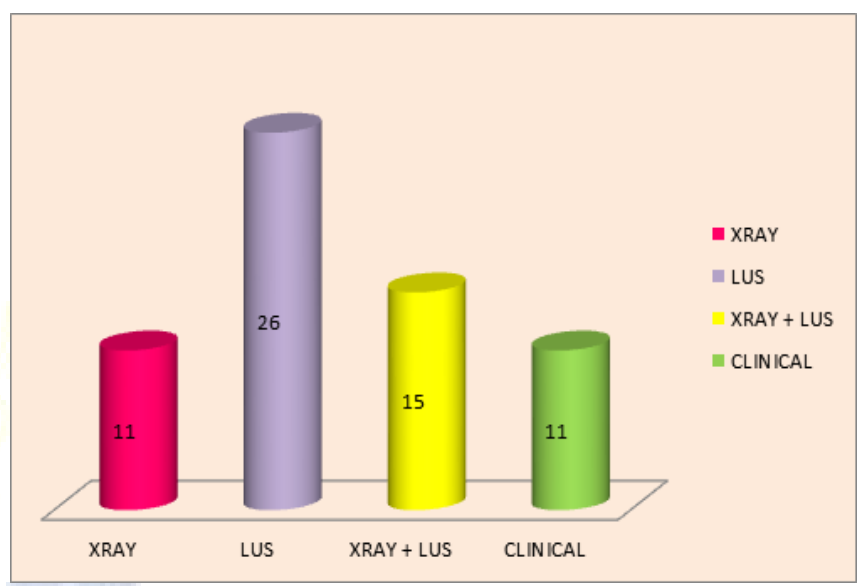

Figure 2: Diagnosis of TTNB by different modalities

[Figure 2] shows the number of TTNB cases diagnosed via different modalities. It has been noted that in 26 cases were diagnosed only via LUS, followed by 15 cases were diagnosed based on x-ray and LUS finding. In 11 cases no features suggestive of TTNB in x-ray and LUS and were diagnosed clinically. P value of 1.00 E-06 noted.

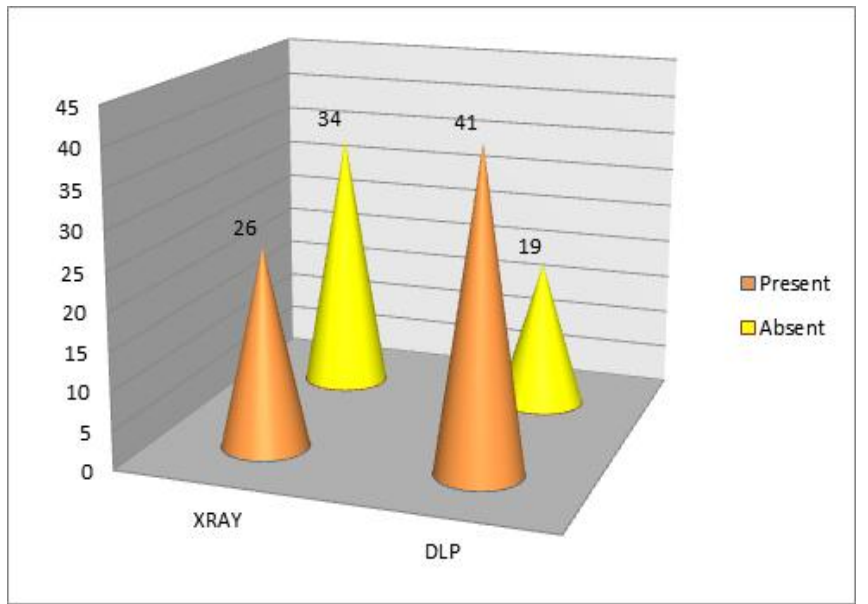

Figure 3: Comparison between x-ray \& DLP in cases of TTNB

[Figure 3] shows percentage of features suggestive of TTNB in x-ray \& USG (DLP). Out of 60 cases of TTNB 26 cases CXR was suggestive of TTNB whereas 34 had no features 
in CXR. At same time on screening via LUS, 41 cases had DLP whereas 19 had no DLP. P value of 0.001 noted. (Fisher's exact test for count data)

\section{LUS \& X-ray manifestations of TTNB}

All the 60 newborns with TTNB on 1st ultrasound evaluation showed either interstitial lung syndrome or pleural line abnormalities with disappearance of A lines.

Out of total cases of TTNB, Double lung point (DLP) was noticed in 41 cases $(68.3 \%)$ and 19 cases(31.6\%) had no DLP which is depicted as very compact B lines in the inferior pulmonary fields while in the superior fields the B lines were present but not compact. This finding was evident in both lungs, though not always symmetrically.

\section{The X-ray Findings considered:}

TTNB:

- Lung hyperinflation/over aeration

- Prominent perihilar interstitial marking (sunburst appearance)

\section{The LUS findings considered:}

- Double lung point (DLP)

- Consolidation with air bronchograms

- Pleural line abnormalities

- Interstitial lung syndrome

- White out lung(compact B lines)

- A line disappearance

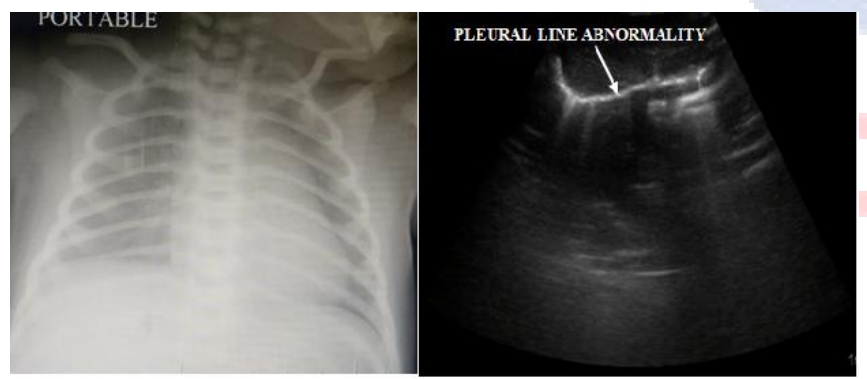

Figure 4: ultrasound manifestation of TTNB: pleural line thickness with disappearance of $A$ lines. The newborn was born via LSCS at gestational age of 35 weeks +3 days. Had respiratory distress at birth. At 2 hours of life CXR was insignificant, however LUS showed pleural line abnormality and disappearance of a lines .No DLP noted.

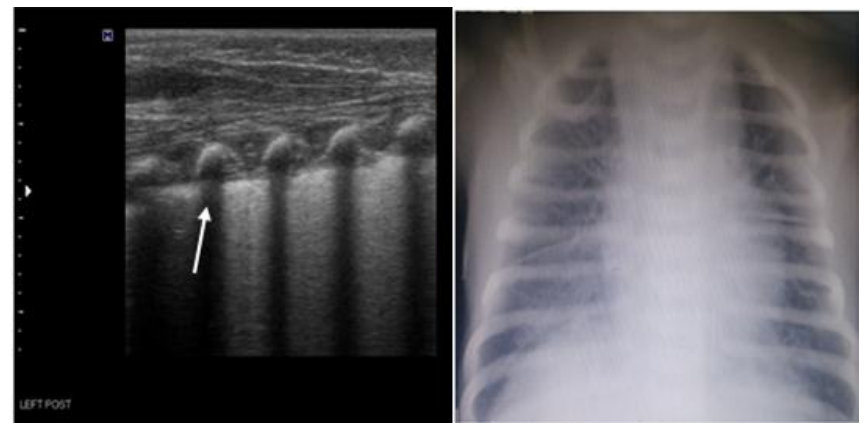

Figure 5: LUS \& X-ray manifestation of TTNB. These are the figures of 40 weeks +2 days gestational age male baby born via
LSCS and noted to have respiratory distress at birth. CXR and LUS done at 3 hours of life. CXR: sunburst appearance with pleural fissure thickening noted bilaterally. LUS showed DLP in posterior regions

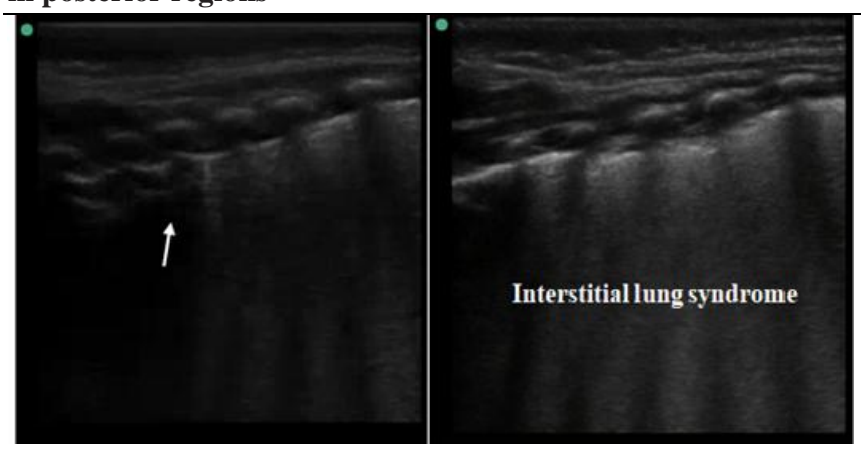

Figure 6: LUS manifestation of 33 weeks old female baby born via NVD. Noted to have tachypnoea at birth. The right lung showed DLP, however DLP was absent on left lung, interstial lung syndrome noted.

\section{Discussion}

This present study was done to define ultrasonographic appearance of TTNB and evaluate its clinical relevance for early diagnosis.

In study by Roberto Copetti et al, ${ }^{[16]} 32$ TTNB cases were compared with 60 normal, 29 RDS, 6 pneumonia cases in time period of 1 year. Gestational ages ranged from 33 to 36 weeks $(34.2+1.01$ weeks, mean $+/-$ SD) and birth weight from 1,620 to $2,730 \mathrm{~g}(2,119+223$, mean $=/-\mathrm{SD}$.

In our study during the $11 / 2$ year study period, 99 babies with respiratory distress were included in this study (after fulfilling inclusion and exclusion criteria). Based on clinical, xray and LUS finding cases were divided into TTNB (60, 60.6\%); RDS (34, 34.3\%); Pneumonia (congenital: 5, 5.5\%) Gestational age ranged from 26 weeks to 40 weeks+ 2 days with mean of $35.6+3.4$ (SD). Birth weight ranged from 785 $\mathrm{g}$ to $4,165 \mathrm{~g}$ with mean of $2,460+810$ (SD). In study by Jing lui et al a statistical significance was noted pertaining to the gestational age, mode of delivery and TTNB however in our study, though most babies with TTNB were term and delivered via LSCS but statistically were insignificant.

In our study TTNB was diagnosed by LUS in gestational age as early 34 weeks which is usually not considered when being evaluated clinically.

Agni et al, ${ }^{[6,7]}$ studied used ultrasonography to diagnose various respiratory conditions and to predict the development of bronchopulmonary dysplasia only via abdominal approach and never used LUS for evaluation. However newer studies are considering the various regions of lung during ultrasound and diagnosis is made based on LUS finding than transabdominal scan of lung field.

In our study longitudinal scan of lung bilaterally was done and compared and a diagnosis was made. 6 lung fields on each side were taken into consideration.

In study by Jing lui et al, ${ }^{[15]}$ TTNB cases were compared 
with normal babies LUS and LUS of RDS cases. It was noted that DLP was noted only in case of TTNB and not in any other case. However other features like a line disappearance, consolidation, pleural line abnormalities were seen in all cases. Similarly in our study we noticed DLP was noted only in TTNB cases. This confirms that DLP sign is specific of TTNB and a great diagnostic marker of TTNB. But consolidation was not seen in any of the TTNB cases.

Studies are lacking pertaining to the comparison of $\mathrm{x}$-ray and LUS .In present study X-ray and LUS of each case was compared at presentation. It was noted that

Robert copetti et al study, sensitivity and specificity of DLP in LUS were noted to be $100 \%$ each respectively.

In study conducted by Jing lui et al sensitivity and specificity of DLP was 76.7 and $100 \%$ respectively.

However in our study sensitivity \& specificity of DLP was $68 \%$ and $100 \%$ suggesting it is more useful as a diagnostic tool than screening. The positive predictive value was noted to be $100 \%$ which indicates that all cases with DLP are diagnostic of TTNB.

In an observational study by Shui Wen Chen at al, ${ }^{[9]} 3405$ babies admitted to NICU irrespective of the cause were screened using LUS and divided into respiratory and non respiratory cases based on LUS.

However in our study we have included cases with respiratory distress and diagnosed further based on LUS finding.

It was also discovered in this study that the ultrasonic features of different lung fields could be different in patients with TTNB. DLP was manifested on one side and interstitial lung syndrome or white lung on the other side; this phenomenon indicated that the water content of the lung tissue (i.e., the degree of pulmonary edema) in different regions was inconsistent in patients with TTNB. Therefore, LUS is beneficial for further understanding lung diseases such as TTNB.

\section{Conclusion}

As it is an inexpensive, easily available bedside tool, it can be routinely used for early diagnosis of TTNB. The easy access, less exposure to radiation and possibility of repeated usage in comparison to Xray makes it more reliable option than Xray

LUS has better specificity than Xray in diagnosis of TTNB. Hence this study demonstrates that LUS can accurately and reliably diagnose TTNB. It can be differentiated easily from RDS and other causes of distress as DLP is seen only in cases of TTNB .Hence, LUS is valuable for distinguishing among causes of neonatal dyspnoea, especially for the differentiation of TTNB and RDS

Therefore LUS could be used widely in NICU as 1st line of diagnostic medium in diagnosis of TTNB for effective management.

\section{Acknowledgment:}

We thank all those who have helped to make this research possible, especially the children, the parents, and the families. Special thanks to Dr. Shwetha, Dr.Sahana ,Dr. Bhaskar and Dr.Shrishail for their invaluable support.We also thank the Staff of department of paediatrics, A J Institute of medical sciences, Mangalore who were involved in the care of these children

\section{References}

1. McGillick EV, Lee K, Yamaoka S, te Pas AB, Crossley KJ, Wallace MJ, Kitchen MJ, Lewis RA, Kerr LT, DeKoninck P, Dekker J. Elevated airway liquid volumes at birth: a potential cause of transient tachypnea of the newborn. Journal of Applied Physiology. 2017 Aug 3;123(5):1204-13.

2. Hooper SB, Harding R. Fetal lung liquid: a major determinant of the growth and functional development of the fetal lung. Clin Exp Pharmacol Physiol 22: 235-241, 1995. doi:10.1111/j.14401681.1995.tb01988.x

3. Jain L, Dudell GG. Respiratory transition in infants delivered by caesarean section. Semin Perinatol 30: 296-304, 2006. doi:10.1053/j.semperi.2006. 07.011.

4. Jain L, Eaton DC. Physiology of fetal lung fluid clearance and the effect of labor. Semin Perinatol 30: 34-43, 2006. doi:10.1053/j.semperi.2006. 01.006.

5. Gomella TL, Cunningham MD, Eyal FG, Tuttle DJ, editors. Neonatology: management, procedures, on-call problems, diseases, and drugs

6. Avni EF, Braude P, Pardue A, Matos C: Hyaline membrane disease in the newborn: diagnosis by ultrasound. Pediatr Radiol 1990; 20: 143146.19

7. Avni EF, Cassart M, de Maertelaer V, Rypens F, Vermeylen D, Gevenois PA: Sonographic prediction of chronic lung disease in the premature undergoing mechanical ventilation. Pediatr Radiol 1996; 26: 463-469

8. Pieper CH, Smith J, Brand EJ: The value of ultrasound examination of the lungs in predicting bronchopulmonary dysplasia. Pediatric Radiol 2004; 34: 227-231.

9. Chen SW, Fu W, Liu J, Wang Y. Routine application of lung ultrasonography in the neonatal intensive care unit. Medicine. 2017 Jan;96(2).

10. Gomella TL, Cunningham MD, Eyal F. Transient tachypnea of the newborn. Neonatology: Management, procedures, on-call problems, diseases and drugs. 7th ed. Stanford: Lange. 2013:919-925

11. Cloherty JP, Eichenwald EC, Stark AR, editors. Manual of neonatal care. Lippincott Williams \& Wilkins; 8th ed. 381

12. Dehdashtian M, Aletayeb M, Malakian A, Aramesh MR, Malvandi H. Clinical course in infants diagnosed with transient tachypnea of newborn: A clinical trial assessing the role of conservative versus conventional management. Journal of the Chinese Medical Association. 2018 Feb 1;81(2):183-6

13. Lokesh Guglani, Satyan Lakshminrusimha, Rita M. Ryan Transient Tachypnea of the Newborn. Pediatrics in Review Nov 2008, 29 (11) e59-e65; DOI: 10.1542/pir.29-11-e59

14. Sperandeo M, Rea G, Santantonio A, Carnevale V. Lung Ultrasonography in Diagnosis of Transient Tachypnea of the Newborn: Limitations and Pitfalls. Chest. 2016 Oct 1;150(4):977-8

15. Liu J, Wang Y, Fu W, Yang CS, Huang JJ. Diagnosis of neonatal transient tachypnea and its differentiation from respiratory distress syndrome using lung ultrasound. Medicine. 2014 Dec;93(27)

16. Copetti R, Cattarossi L. The 'double lung point': an ultrasound sign diagnostic of transient tachypnea of the newborn. Neonatology. 2007;91(3):203-9. 
Copyright: () the author(s), 2020. It is an open-access article distributed under the terms of the Creative Commons Attribution License (CC BY 4.0), which permits authors to retain ownership of the copyright for their content, and allow anyone to download, reuse, reprint, modify, distribute and/or copy the content as long as the original authors and source are cited.

How to cite this article: Poorvi, Oommen RA, Soans ST. Double Lung Point Vs X-Ray in Transient Tachypnea of Newborn. Asian J. Clin. Pediatr. Neonatol.2020;8(1):52-57.

DOI: dx.doi.org/10.47009/ajcpn.2020.8.1.13

Source of Support: Nil, Conflict of Interest: None declared. 